\title{
Overview of Missile Control Method
}

\author{
Chen Li, Jingyu Yang, Qing Wang, Zhijie Zhang, Hailong Wang, Jiahe Sun, Tianfen Mo and Shiwei Sun \\ Laboratory of Space Solar Power Station Dynamics and Control, Innovation Studio of Marine-Space Science \& Technology, \\ Faculty of Aerospace Engineering, Shenyang Aerospace University, Shenyang, China
}

\begin{abstract}
The classical guidance law and the modern guidance law in the missile control system are introduced comparatively comprehensive. Firstly, the classical guidance law is introduced; Then the guidance law based on modern control theory is introduced emphatically, and the advantages and disadvantages of these guidance laws and development trend are analyzed. Finally, several kinds of composite control methods are expounded briefly.
\end{abstract}

Keywords-classical guidance law; missile control method; modern guidance law

\section{INTRODUCTION}

Missile is a kind of aircraft which carries warhead, relys its own power device to fly and it is guided by the guidance system to control the flight path, it is a modern high-tech weapon system, its main task is to target the implementation of precision to combat and interception. Guidance system is the most important part of the missile structure, meanwhile missile guidance law is a crucial part of the guidance system and the core of the entire guidance system. The guidance law determines the missile's center of mass movement and affects the missile's interception effect directly. This paper introduces the classical guidance law and the guidance law based on modern control theory.

\section{Classical GUIDANCE LAW[1]}

The guidance laws based on early concepts are commonly referred to as classical guidance laws, all of the classical guidance law is deduced from different battlefield environment and approach to the target with high precision and rapid. At present, most active missiles in actual combat still use classical guidance law, because it has many advantages such as requiring less information of target, less requirement for the measuring element, simple form of the guidance law and easy to implement. The classical guidance law mainly includes tracking method, constant bearing navigation, three-point guidance, front method, proportional navigation method.

\section{A. Tracking Method}

Tracking method is a guidance method the missile in the process of flying to the target whose speed vector always points to the target. This guidance method requires that the angle between the missile velocity vector and the target line of sight (front angle) is always equal to zero. This method is proposed earlier, it has advantages like easily to realize, the technical difficulty is small. However, the ballistic characteristics of this method have obvious disadvantages. In this guided mode, the absolute speed of the missile always points to the target, resulting in the relative speed of the missile target has been lagging behind the missile target line of sight, the missile always needs to fly to the target directly behind. Missile flight trajectory is more curved, requiring a larger normal overload, so it requires missile has a higher mobility. Therefore, tracking method applied not much.

\section{B. Constant Bearing Navigation}

To overcome the shortcomings of tracking method that the relative speed is behind the target line of sight and the missile need revolve to the rear of target to attack, constant bearing Navigation was proposed. Missiles' line of sight has always maintained parallel movement in the process of intercepting the target. At the same time, the line-of-sight angular rate is always kept at zero. Parallel approximation method is an ideal guidance method. The advantages are obvious, for example the ballistic is smooth based on parallel approach, the missile normal overload is small, the maneuvering target missile required for normal overload is less than the target maneuver. However, this method is difficult to achieve engineering requirements on the guidance system. It requires accurate acquisition of missile and target status information and strictly and accurately implement the guidance program.

\section{Three-Point Guidance}

The three-point method is that the missile is always in the guidance station and the target connection during the whole guidance flight, this guidance is mainly used for remote guidance. The guidance station may be either fixed or movable. This guidance way is simply to achieve and has great ability of Anti-jamming. Its shortcomings are ballistic bending, poor maneuverability to cope with targets, needing more overload, overload easily to saturate. In the use of such guidance to intercept low-altitude target missile trajectory will appear sink phenomenon.

\section{Front Method}

This method makes the missile ahead of the target line of sight of an angle in the target flight direction, also known as the angle method or straightening method. The guidance law makes the missile trajectory straight, at the hit point of the overload is less and less affected by the target maneuver. But the guidance parameter is too much, the system structure is complex, anti-jamming ability is bad, is not conducive to the project realization.

\section{E. Proportional Navigation Method[2]}

Proportional guidance method refers to the angular velocity of the velocity vector of the missile is proportional to the rotational angular velocity of the line of sight in the process of missile intercept target. Proportional guidance law 
is widely used because of its simple form, less demand information, smooth trajectory, and easy implementation. Proportional guidance law has many forms such as pure proportional navigation law (PPN), true proportional navigation law (TPN), generalized proportional navigation law (GPN) and so an. Although there are many advantages of proportional guidance law, the large maneuvering or rapid maneuvering target will still have a larger miss distance.

\section{GUIDANCE LAW BASED ON MODERN CONTROL THEORY}

With the rapid development of modern military technology, complex air combat environment for intercepting high-speed mobile target, or target using a variety of interference and defensive measures, the use of Proportional Navigation Method is difficult to meet the requirements of precision, so the classical guidance law appears powerless. Therefore, in order to adapt to the modern complex combat environment, it is urgent to study the new modern guidance law.

Compared with the classical control method, the modern control method can effectively suppress the influence of parameter perturbation and various disturbances, and can track the unmodeled dynamics of the system, and thus has better control performance. This makes the modern control method widely used in the design of missile control system, which greatly promoted the development of missile flight control technology.

\section{A. Optimal Guidance Law [3]}

Optimal control was first applied in the design of guidance law in the 1960s. Optimal guidance law performance index function generally select a weighted combination of intercept time and control for the miss distance and the relative motion equation of missile and target and some other requirements can be used as constraint conditions, the principle of optimal guidance law can be launched by the minimum value. At present, the optimal guidance law is studied based on linear quadratic Gaussian (LQG) theory, and it has entered the practical stage. However, the structure of the optimal guidance law is more, and the motion information of the target is designed under the known conditions, which requires a high requirement for the missile's measuring element. At the same time, in the process of the missile tracking target, it is needed to estimate the remaining flight time in real time. When the estimation error is large, the guidance precision of the missile decreases sharply.

\section{B. Sliding Mode Variable Structure Control[4]}

The main idea of variable structure control is to use the high speed switching control law, the state trajectory of the drive system asymptotically reaches a pre designed state space surface, so as to ensure the robustness of the system to the external perturbation. Sliding mode variable structure control is an important nonlinear control method, which has the advantages of fast response, small overshoot, simple structure, and strong robustness to model uncertainty and external disturbance, so it is widely used in the design of flight control system.

Although the variable structure has good adaptability and robustness in theory, the variable structure control law needs the upper bound of the known system uncertainty in the design process, so the design is conservative and easy to cause the control the saturation problem. In addition, due to the inertia of the switching device, the frequent switching of the variable structure system in different control logic, the actual system is easy to cause the system's violent chattering, which can cause great harm to the actual system. Therefore, it is an important subject of variable structure control system design to weaken the chattering without affecting the robustness.

\section{Guidance Law Based on Robust Control[5]}

Robust control is an important means to solve the problem of system uncertainty, which has been developing rapidly since the 1980s. The core idea is to design a controller with a fixed parameter after the range of the known object, so as to ensure that the dynamic performance of the object satisfies certain indexes when the object is perturbed in this range.

As the robust control theory can effectively suppress the parameter uncertainties and external disturbances in nonlinear systems, the guidance law based on the robust control theory design has been widely developed. There are $\mathrm{H}_{\infty}$ robust guidance law, fuzzy $\mathrm{H}_{\infty}$ guidance law and $\mathrm{H}_{2}, \mathrm{H}_{\infty}$ robust guidance law based on control theory. However, the guidance law based on control theory needs to solve the Hamilton-Jacobi partial differential inequality (HJPDI), and the analytic solution of HJPDI is more difficult. In the process

of implementing the $\mathrm{H}_{\infty}$ nonlinear guidance law, it is required that the longitudinal velocity of the missile must be controllable, and this requirement is difficult to satisfy in many actual combat.

\section{CONCLUDING REMARKS}

The increasingly complex battlefield environment and the increasing target flight characteristics of modern warfare place a higher demand on the technical and tactical targets of various missiles. Therefore, the development of missile control technology is getting more and more attention. It is a very necessary work to study the advanced guidance law with better performance and higher precision. It is believed that through the continuous efforts of people and the comprehensive development of modern science and technology, Multi-advanced guidance law will be designed.

\section{ACKNOWLEDGMENT}

This paper is supported by the innovation and Entrepreneurship Program of the University of Aeronautics and Astronautics in Shenyang (Grant No.DX501312).This project is supported by National Natural Science Foundation of China (Grant No. 51605308) "Co-evolution of fractional order coupled structure vibration-attitude dynamic and control of spacecraft"; The Liaoning Province doctor Science Research Fund Project "Co-evolution of fractional order coupled structure vibration-attitude dynamic and control of China Space Station"(Grant No. 201601178) ; The Liaoning Province "13th Five Year Plan" Higher Education Research Fund Project "Teaching quality monitoring system and 
safeguard mechanism research on Aerospace engineering majors” (Grant No. GHZD160012); The Liaoning Province Department of Education Fund Project "Study of fractional order coupled structure vibration-attitude dynamic and control of spacecraft” (Grant No. L2015414); The Liaoning Province Department of Education Fund Project "Research and practice on specialty of aerospace engineering based on recognition for engineering education”(Grant No.030201619); "Exploration and Practice of 'Theory+ Interesting+ Research' Innovative Teaching Mode”(Grant No.YJS2014-11); "Research on Intelligent Integrated Control of Coupling between Space Solar Power Station Structure Vibration and Attitude Control (Grant No.13YB22)"; "Simulation and experimental platform design of virtual control of hypersonic aircrafts (Grant No.DX501312)"; "Health monitoring system design of attitude-structure vibration of in-orbit spacecraft" (Grant No. 201610143050); "Emote operation, display and control of electromagnetic launcher(Grant No. X1610318)”; “Design and analysis of intelligent metamaterial cloaking structure system (Grant No. X1610317)”.

\section{REFERENCE}

[1] Classical and fuzzy-system guidance laws in homing missiles systems. (2016), 34722

[2] Chen, Y. Y. (2016). Robust terminal guidance law design for missiles against maneuvering targets. Aerospace Science and Technology, 54, 198-207. https://doi.org/10.1016/j.ast.2016.03.028

[3] Wang, H., Lin, D., Cheng, Z., \& Wang, J. (2014). Optimal guidance of extended trajectory shaping. Chinese Journal of Aeronautics, 27(5), 1259-1272. https://doi.org/10.1016/j.cja.2014.03.022

[4] Ran, M., Wang, Q., Hou, D., \& Dong, C. (2014). Backstepping design of missile guidance and control based on adaptive fuzzy sliding mode control. Chinese Journal of Aeronautics, 27(3), 634-642. https://doi.org/10.1016/j.cja.2014.04.007

[5] Zhou, D., \& Li, Q. (2014). Indirect robust control of agile missile via Theta-D technique. Defence Technology, 10(3), 269-278. https://doi.org/10.1016/j.dt.2014.06.009 